\title{
High Speed X-Ray Imaging for Nozzle Exit Velocity and Density Distribution Measurements of GDI Nozzles
}

\author{
Raphael Distler ${ }^{1}$, Dr. Christoph Hamann ${ }^{1}$, Dr. Martin Krämer ${ }^{1}$, Dr. Eberhard Kull ${ }^{1}$, Prof. Dr. \\ Michael Wensing ${ }^{2}$, Dr. Zhilong $\mathrm{Li}^{3}, \mathrm{Ya} \mathrm{Gao}^{3}$, Dr. Jin Wang ${ }^{3}$ \\ ${ }^{1}$ Continental Automotive $\mathrm{GmbH}$, Regensburg, Germany \\ ${ }^{2}$ Lehrstuhl für technische Thermodynamik, Friedrich-Alexander-Universität \\ Erlangen-Nürnberg, Germany \\ ${ }^{3}$ Advanced Photon Source, Argonne National Laboratory, United States of America \\ *Corresponding author: raphael.distler-ext@continental-corporation.com
}

\begin{abstract}
Investigation of the primary breakup region of gasoline sprays is important for future nozzle development. It improves the principal understanding of inner nozzle flow and spray breakup. It also allows validating and developing CFD models. Due to the high optical density common measurement techniques like Phase Doppler Anemometry reach their limit in optical dense sprays as in the primary breakup region. High Speed X-Ray Imaging is capable to measure 2D velocity distributions directly at the spray hole exit. For generating the intense X-Ray beam the synchrotron Advanced Photon Source at Argonne National Laboratory is used. Passing through the spray the X-Ray beam is changed by two different physical principles: absorption and phase contrast. Absorption can be applied to measure the density of the spray. Phase contrast is used to visualize the borders of droplets and ligaments with high contrast. The accelerated electron bunches inside the synchrotron have a constant period length to each other. This leads to an accurate pulsed X-Ray beam (periodicity: $68 \mathrm{~ns}$ ). The use of multi exposure with very short X-Ray pulses (17 ns) shows the traveled distance of the spray droplets and ligaments. The spray speed $(150-250 \mathrm{~m} / \mathrm{s})$ is calculated by dividing these distances with the time gap between two X-Ray pulses. The X-Ray measured density distributions and velocity distributions are combined to calculate the spray force rate. The so gained force rate is validated with a spray force measurement performed at the Spray Momentum Test Bench (SMTB) at Continental Automotive $\mathrm{GmbH}$. The study is focusing on the measurement setup of High Speed X-Ray Imaging at Argonne National Laboratory and the evaluation algorithms.
\end{abstract}

\section{Keywords}

High Speed X-Ray, Gasoline Direct Injection, Gasoline Spray, Primary Breakup, Spray Density, Spray Velocity

\section{Introduction}

The spray performance of a GDI injector is highly influencing the efficiency and pollutant emissions of modern gasoline combustion engines. To optimize the whole process from injection over mixture formation and the final combustion, the behavior of a single spray plume has to be understood. To find a direct link between nozzle geometry and generated spray, the primary breakup region has to be analyzed. The primary spray velocity and density distribution are the key factors for further spray breakup. Measurement techniques like Phase Doppler Anemometry and Laser Induced Fluorescence reach their limit in optical dense sprays as in the primary breakup region. Therefore no information about the velocity and density distribution at the nozzle exit is available for comparison of nozzles and as input for CFD simulations. High Speed X-Ray Imaging is able to capture both. Out of the density and velocity distributions, the spray momentum can be calculated and for verification compared with a mechanically measured spray momentum distribution. $[1,2,3,4]$

\section{Hydraulic Injector and Spray Parameters}

The correlation between massflow, flow velocity and resulting momentum can be theoretically calculated by the law of Bernoulli. Equation 1 gives the theoretically massflow $\dot{m}_{t h}$ depending on the flow cross section $A$ the fuel density $\rho$ and the relative fuel pressure $\Delta p$. Equation 2 shows the correlation between the flow speed $v_{t h}$, the relative fuel pressure $\Delta p$ and the fuel density $\rho$. [5]

$$
\begin{aligned}
& m_{t h}=A v \rho=A \sqrt{2 \rho \Delta p} \\
& v_{t h}=\sqrt{\frac{2 \Delta p}{\rho}}
\end{aligned}
$$

Due to phenomena like turbulence and cavitation, the flow velocity and massflow in real nozzles is smaller. For this reason different types of discharge coefficients are defined. $C_{D}$ is giving the ratio of the effective massflow 
$\dot{m}_{\text {eff }}$ to the theoretically calculated massflow $\dot{m}_{t h}$. (Equation 3) The $C_{A}$ is defining the ratio of the effective used hydraulic flow cross section $A_{\text {eff }}$ related to the theoretical flow cross section $A_{0}$. (Equation 4) This equation is directly linked to the model of the vena contracta. The ratio of the theoretical flow velocity to the real flow velocity is given by Equation 5. The product of $C_{V}$ and $C_{A}$ delivers the $C_{D}$. (Equation 6) [5]

$$
\begin{aligned}
& C_{D}=\frac{\dot{m}_{e f f}}{\dot{m}_{t h}} \\
& C_{A}=\frac{A_{e f f}}{A_{0}} \\
& C_{v}=\frac{v_{e f f}}{v_{t h}} \\
& C_{D}=C_{A} \cdot C_{v}
\end{aligned}
$$

By adding these discharge coefficients to the Bernoulli equations the following correlations are developed. (Equation 7, Equation 8) [5]

$$
\begin{aligned}
& m_{\dot{e f f}}=C_{D} A_{0} \sqrt{2 \rho \Delta p} \\
& v_{\text {eff }}=C_{v} \sqrt{\frac{2 \Delta p}{\rho}}=C_{D} \frac{A_{0}}{A_{\text {eff }}} \sqrt{\frac{2 \Delta p}{\rho}}
\end{aligned}
$$

By multiplication of the massflow and the velocity the momentum flow $\dot{p}_{e f f}$ is calculated. (Equation 9) [5]

$$
\dot{p}_{e f f}=C_{D}^{2} \frac{A_{0}^{2}}{A_{e f f}} 2 p
$$

\section{Basics on X-Ray Radiation and its Interaction with Fuel}

Hard X-Ray radiation describes light with a wavelength from $<5-250 \mathrm{pm}$ which corresponds to a photon energy of $5-250 \mathrm{keV}$. For the measurements at ANL X-Ray radiation with a photon energy of up to $9 \mathrm{keV}$ is used. X-Ray radiation can be produced in different ways: [6]

- highly energetic transients in electron shells of atoms or molecules: In X-Ray tubes a cathode is producing free electrons. These electrons are accelerated by an electromagnetic field towards a target. If a free electron has enough energy and pushes out an orbital electron of an atom in the target, by hitting the atom, the vacancy of the left electron has to be filled up with an higher energetic electron. While filling up the vacancy, the higher energetic electron has to emit a photon to reach the correct energy level. This photon is the produced X-Ray photon and has a material characteristic wavelength.

- deceleration of charged particles like electrons and positive ions: Particles which are getting deflected by an electrical field send out so called Bremsstrahlung: This effect delivers X-Ray radiation with a continuous spectrum.

- synchrotron radiation is a special type of Bremsstrahlung: In a synchrotron charged particles are bent at every edge of the ring. This bent equals a deceleration of the particle in one direction while acceleration in another direction. A consequence of this is Bremsstrahlung. Undulators and wigglers are used for adding oscillations on the electron bunches and to produce synchrotron radiation with a broad spectrum. As the electron bunches in a synchrotron are highly collimated, a very intensive and collimated beam can be produced. The radiation is linearly or circularly polarized.

The used synchrotron, the Advanced Photon Source (APS) at the Argonne National Laboratory, is providing a special running mode: the hybrid-singled mode. Figure 1 illustrates the used measurement setup. In the APS electron storage ring with its perimeter of $1104 \mathrm{~m}$, nine bunches of electrons are circulating in a ring with nearly the velocity of light. This results in a running frequency of $274 \mathrm{kHz}$. Eight of the nine bunches build up a so called bunch train. The ninth bunch with a current of $16 \mathrm{~mA}$ is brighter than the single bunches in the bunch train with a current of $11 \mathrm{~mA}$ each. The periodicity of the bunches of the bunch train is $68 \mathrm{~ns}$. The overall bunch train duration is $500 \mathrm{~ns}$. The duration of each bunch is $17 \mathrm{~ns}$. [7] The time gap between the super bunch and the bunch train is in each direction $1.569 \mu \mathrm{s}$. When one bunch is passing the undulator of the used beamline, X-Ray radiation is decoupled into the laboratory. The produced X-Ray radiation has a continuous spectrum and is characteristically collimated. [7] A setup of different shutters is used to cut off the not needed X-Ray pulses. The injector is mounted tip down into a spray chamber. The X-Ray pulses are passing and interacting with the spray. The X-Ray images 


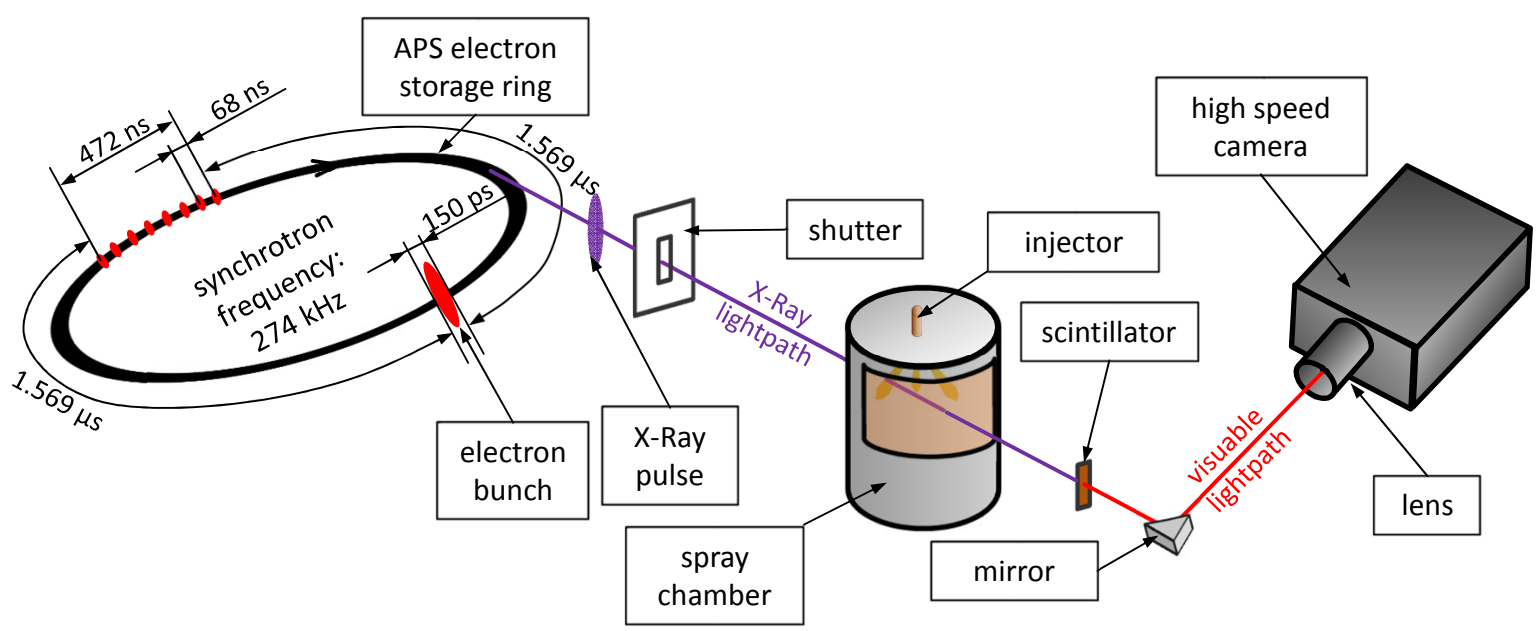

Figure 1. Measurement setup for High Speed X-Ray Imaging at the Advanced Photon Source of the Argonne National Laboratory based on [7]

are converted to visible light images by a fast scintillator. The visible light is reflected into a high speed camera by a mirror. [7]

The interaction of X-Ray radiation with matter can be separated into two different phenomena: absorption and phase contrast. The phenomena of absorption is described by the law of Lambert-Beer-Bonguer. Light looses a constant fraction of its intensity by passing a constant material thickness. This results in a exponentially relationship between the light intensity $(I(x))$ and the material thickness the light passed through. $I(x)$ is calculated by its material specific and wavelength depended absorption coefficient $\alpha$ and the passing distance of the light $x$. (Equation 10) [8]

$$
I(x)=I_{0} e^{-\alpha x}
$$

The second phenomena, the phase contrast, is caused by refraction of a coherent X-Ray beam on phase borders where a gradient in X-Ray refraction indices is occurring. The reflection angle is depended on the X-Ray spectrum's wavelenghts and the difference of the refraction indices. Phase contrast imaging in common is used to separate small structures in material compounds where both materials have very similar absorption coefficients. The refraction leads to high contrast on the image. Its visibility on the captured image is mainly influenced by the distance between the measurement object and the detector, or in the here used setup of the scintillator. Detailed information about phase contrast imaging can be found in [9].

\section{Basics of the Used Measurement Process and Evaluation Algorithms}

For obtaining the velocity of the spray in the near field a multiexposure of the injection is captured by using three $X$ Ray pulses of the described bunch train. By knowing the time interval between the single bunches and by measuring the distance a structure traveled during the exposures, its' velocity can be calculated. To enhance the phase contrast intensity and therefore to visualize the structure edges clearly, a longer distance between spray and scintillator is used. For obtaining the density distribution of the fuel in the spray the effect of phase contrast is minimized by shortening the distance between spray and scintillator and by using an X-Ray mirror inside the beam path to select only low-energy photons at $7 \mathrm{keV}$.

\section{Algorithm for the Spray Velocity Evaluation}

For evaluating the spray velocity a novel algorithm is developed to enhance the spatial and temporal resolution of the velocity result. Like common Particle Image Velocimetry algorithms, it is based on auto correlation of the image. Figure 2 gives an exemplaric overview of the single evaluation steps. The raw image (1) gets background corrected by subtracting an image without any spray. (2) Furthermore the contrast of the image gets enhanced. In (3) the image is divided into 26 by 25 squares and the resolution of the image is enlarged by calculating subpixels. The square size defines the spatial velocity resolution in the final evaluation. A parameter study with synthetic generated velocity images was done to find a suitable velocity resolution without a systematic result distortion. The viable resolution is mainly influenced by the average spray velocity, the pixelsize and the exposure gap. The single squares are getting autocorrelated (4). To identify the realistic peaks from the autocorrelation the image gets rotated that the flow is roughly from upside down. (5) The rotation angle is defined at $30^{\circ}$ (= height angle of the single spray jet). In (6) the autocorrelated image is cropped to the relevant part of the image for the velocity information. As the main peak of an autocorrelation image is always in the middle of the image it is cut to a region which is able to show all 5 peaks of a multiexposed structure with a maximum velocity of $300 \mathrm{~m} / \mathrm{s}$ in axial $(\mathrm{z})$ and $50 \mathrm{~m} / \mathrm{s}(\mathrm{y})$ in radial direction. The experiments shown in this paper are done with $\mathrm{n}$-heptane at 200 bar injection pressure which results in a Bernoulli velocity of $240 \mathrm{~m} / \mathrm{s}$. In a next step the positions of the peaks are getting located. Plotting the row/column position of the maxima against their number and applying straight fits delivers the average slopes, 
which are the corresponding average moved distances the spray passed during two exposures in axial and radial distance. With this information the velocities are calculated. A reversing of the before done image rotation (5) by using basic trigonometry is delivering the correct axial and radial spray velocities. The theorem of Pythagoras is used to calculate the absolute spray velocity.

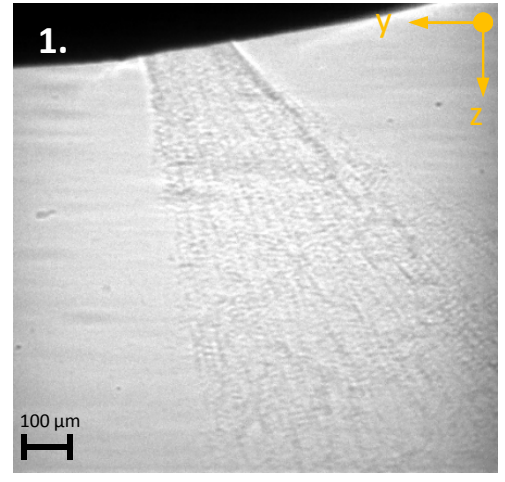

raw image (multi exposure)

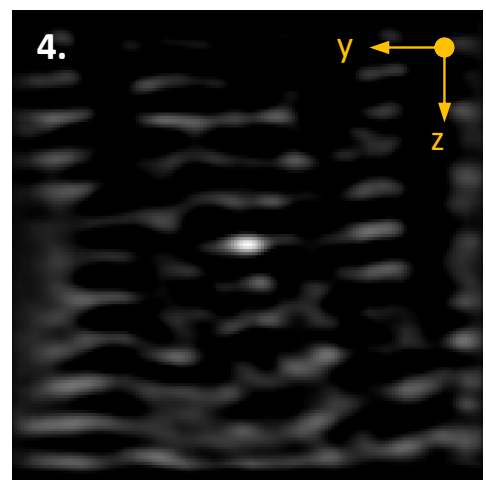

auto correlated image

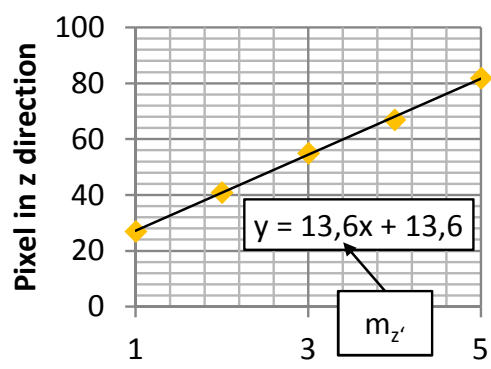

no. of maximum

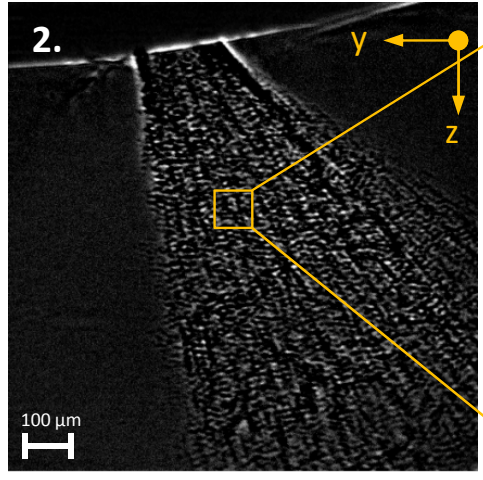

background corrected

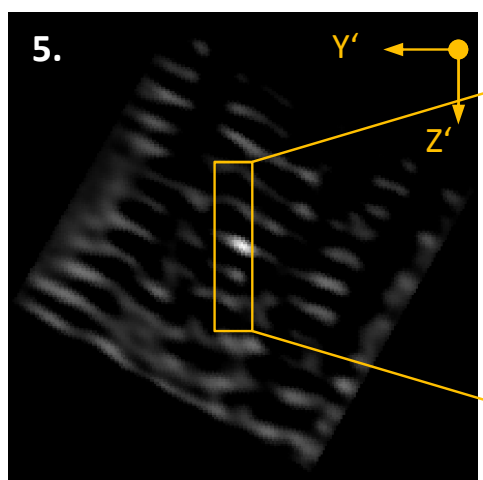

roughly rotated auto correlated image

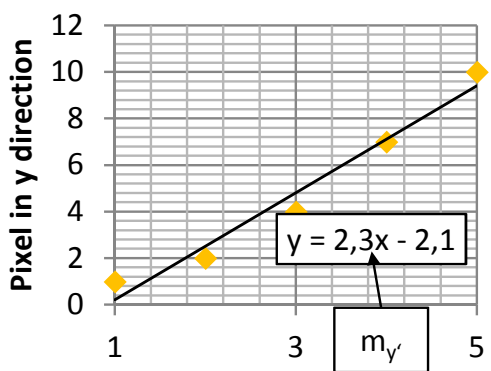

no. of maximum

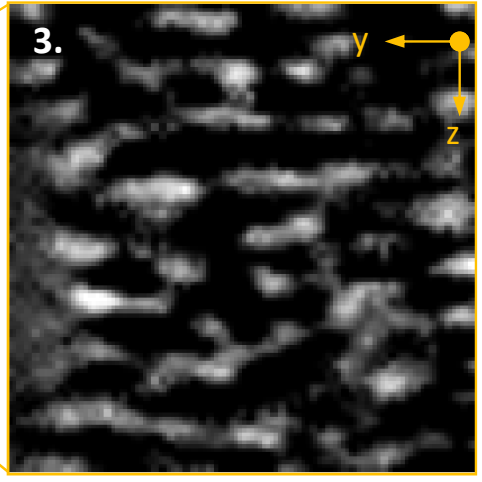

cutting of the image into pieces

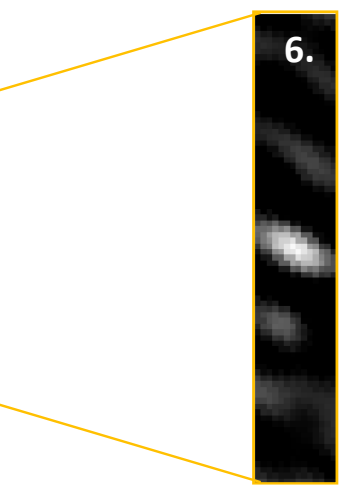

cropped to the relevant image region

$$
\begin{gathered}
v_{z^{\prime}}=m_{z^{\prime}} * \frac{\text { pixelsize }}{\text { exposuregap }} \\
v_{y^{\prime}}=m_{y^{\prime}} * \frac{\text { pixelsize }}{\text { exposuregap }} \\
v_{\text {res }}=\sqrt{v_{z^{\prime}}^{2}+v_{y}^{2}}
\end{gathered}
$$

Figure 2. Basic process of the velocity evaluation algorithm

\section{Algorithm for the Spray Density Evaluation}

Although the effect of phase contrast is minimized by shortening the distance between spray and scintillator, the phase contrast signal still is by order of magnitudes higher than the absorption signal in the image. For this reason the developed density evaluation algorithm has to filter the phase contrast. One basic idea of the spray density evaluation is that the effect of phase contrast is not producing any light, it just leads to a local redistribution of the intensities. Therefore the integrated intensity of the X-Ray is not influenced by the effect of phase contrast. Figure 3 shows the two steps which are used for the density evaluation. The raw image gets background corrected by subtracting an image without any spray. For the filtering of the background corrected image different types of filters and filter settings are analyzed on synthetic density images which are distorted by phase contrast. As a result a median filter is chosen. Also in literature the recommended filter for phase contrast is a median filter. [10]

The filter error has to be corrected by adjusting the overall grey value sum of the image to the grey value sum of the background corrected image.

For calibrating the measurement setup, capillaries of different diameters filled with $n$-heptane are used. By knowing the density of the n-heptane and the size of the capillaries the absorption coefficient of the fuel for the used setup and evaluation calibration can be calculated. Figure 4 shows the X-Ray transmission against thickness of a n-heptane pillar. By applying the law of Lambert Beer-Bonquer the transmission coefficient $\mu$ can be calculated. Applying this 


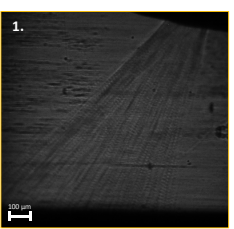

raw image

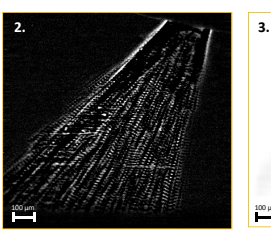

background corrected

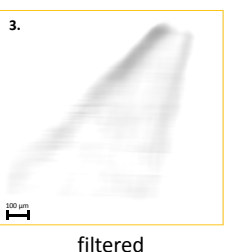

filtered

Figure 3. Basic process of the density evaluation algorithm

coefficient to the image the density distribution inside the spray is delivered. (e.g. Figure 5) The scale can be given in fluid path length and projected spray density. The fluid path length describes how thick a liquid fuel pillar has to be to deliver the same absorption. The projected spray density gives the density distribution integrated in the line of sight.

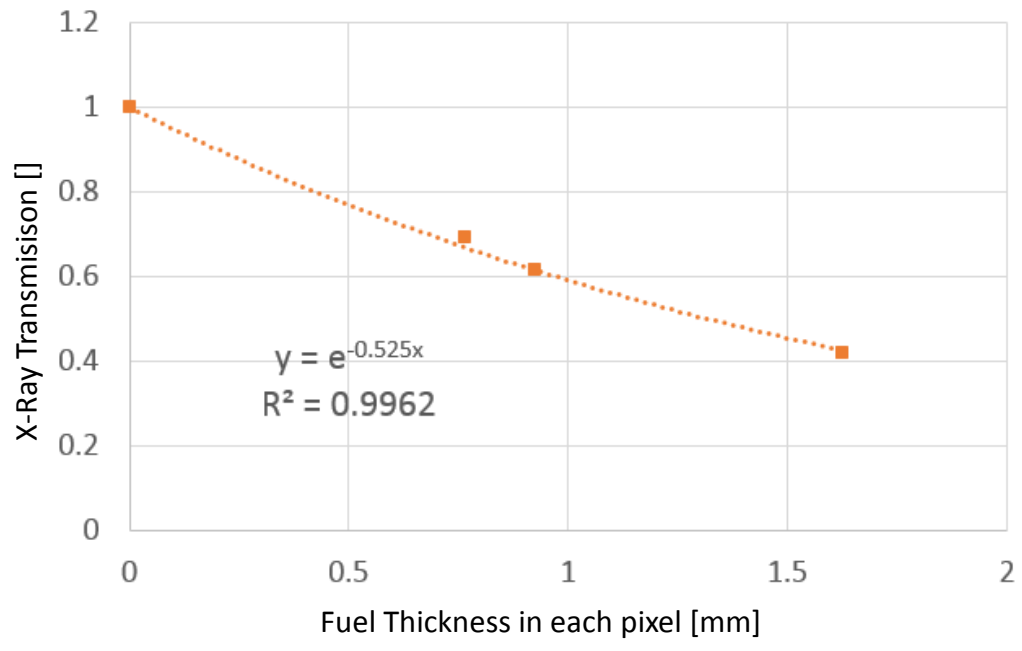

Figure 4. Calibration result of the used measurement setup for different capillaries filled with $\mathrm{n}$-heptane
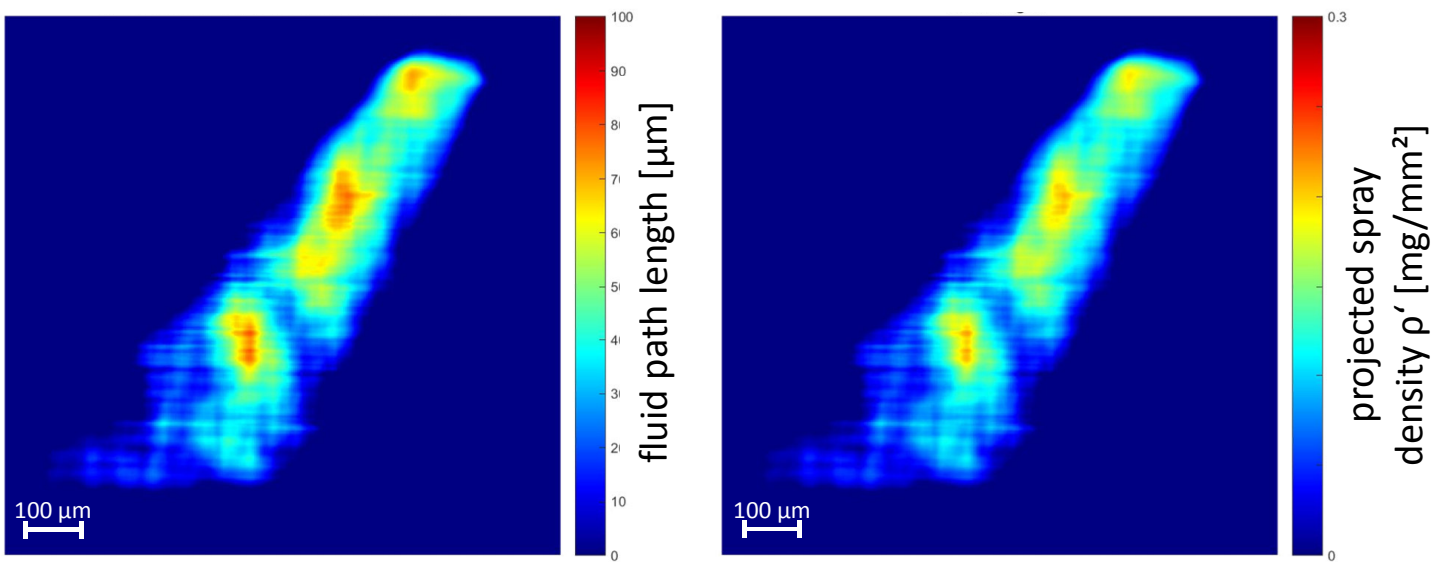

Figure 5. Example for the spray density evaluation, left = fluid path length the X-Ray is passing; right = projected spray density

\section{Calculation of the Momentum out of X-Ray Measurements}

Figure 6 illustrates the information the X-Ray measurement is providing. The velocities and densities are depended on the two spatial coordinates $z$ and $y$ and the point of time $t$. For calculating the spray momentum, spray force and massflow rate, a cut through the spray rectangular to the flow direction is done. For each pixel on this cutting line the spray parameters are getting calculated.

The massflow in $z$ direction is calculated in Equation 11 by the product of the density $\rho^{\prime}(y, z, t)$, the velocity $v_{a b s}(y, z, t)$ and the pixelsize.

$$
\dot{m}_{a b s}(y, t)=\rho^{\prime} \cdot v_{a b s} \cdot l_{\text {pixel }}
$$




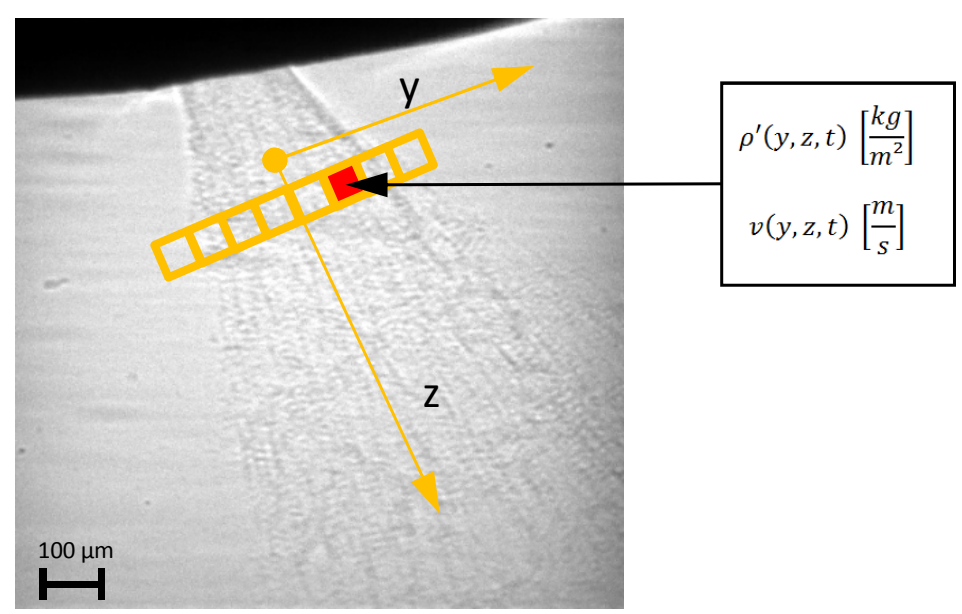

Figure 6. Information provided by the X-Ray measurement

The spray force is calculated by the product of the massflow $\dot{m}_{a b s}(y, t)$ and the velocity $v_{a b s}(y, t)$. (Equation 12)

$$
F_{a b s}(y, t)=\dot{m}_{a b s}(y, t) \cdot v_{a b s}(y, t)
$$

By integrating the spray forces $F_{a b s}(y, t)$ in $y$ direction, the overall spray force rate is calculated. Integrating this force rate over time delivers the spray momentum. (Equation 13)

$$
p_{a b s}=\iint F_{a b s}(y, t) d y d t
$$

\section{Basics of Spray Momentum Measurements}

The measurement principle used on the SMTB is illustrated in Figure 7. A target pin is mounted on a piezoelectric force sensor and can be freely positioned in a spherical coordinate system around the injector tip. As the target pin in the used setup has a diameter of $1 \mathrm{~mm}$ a shielding cap is used to ensure the force sensor only measures the force which is induced on the pin and not the force of spray which is hitting the sensor itself. The target pin surface is always faced to the origin of the spherical coordinate system. Therefore only the axial force of the spray can be measured. Force on the piezoelectric sensor is producing electric charge. This charge gets amplified and converted into a time depended voltage signal which corresponds to a force rate. The force rate has to get filtered at the sensor's critical frequency. By integration of the force rate, the momentum is calculated. As a small pin is used which is not able to capture the whole spray, the momentum of a plume has to be calculated out of several measurement points. A basic theory about the mathematical principle can be found in [5].

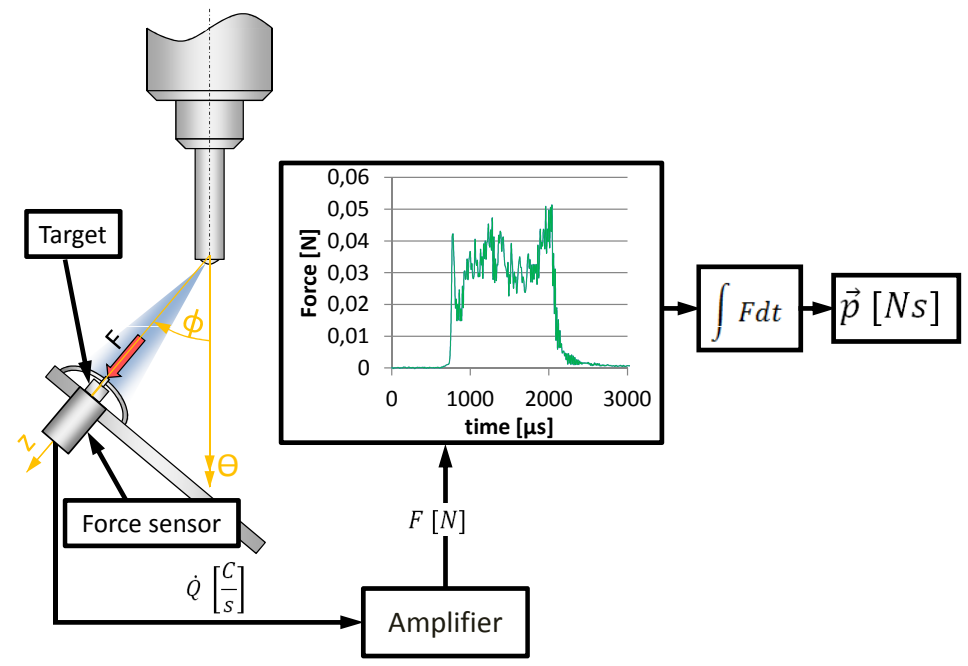

Figure 7. Measurement principle of the SMTB 


\section{Results}

Figure 8 shows an exemplaric mass and velocity rate for a gasoline spray an the distance of $400 \mu m$ to the sprayhole exit. By averaging the rates the discharge coefficients are calculated by using Equation $3\left(C_{D}\right)$, Equation $5\left(C_{V}\right)$ and Equation $6\left(C_{A}\right)$.

$$
\begin{aligned}
& \text { - } C_{v}=0,85 \\
& \text { - } C_{A}=0,65 \\
& \text { - } C_{D}=0,55
\end{aligned}
$$
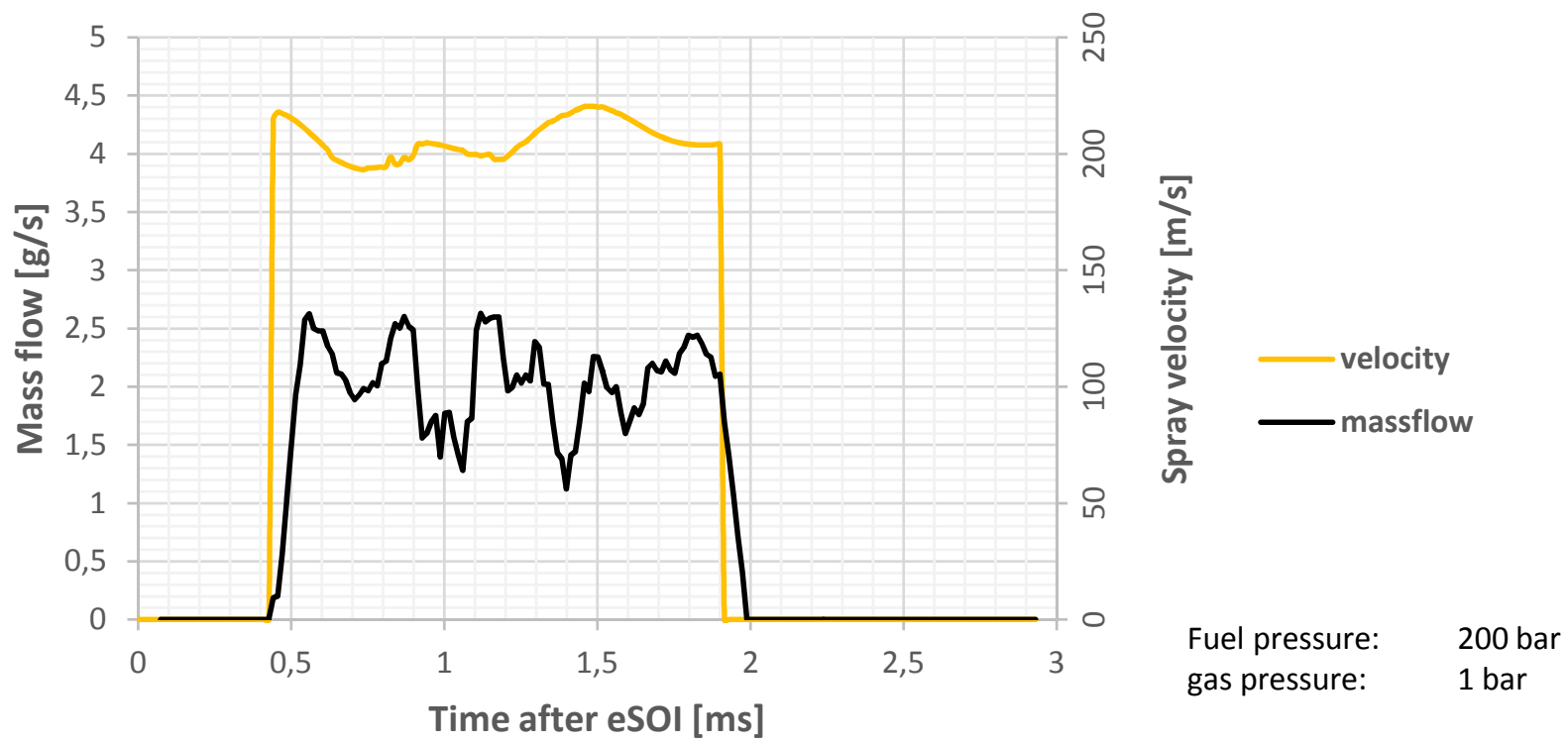

Figure 8. Exemplaric mass and velocity rate measured with High Speed X-Ray Imaging for a Gasoline Spray at 200 bar

For the comparison of the resulting spray force rates and the spray momentum the mechanically captured force rate has to be aligned. The SMTB runs with Exxsol D40. The High Speed X-Ray measurements are performed with $\mathrm{n}$-heptane. For this reason the spray momentum force rate is corrected by the density ratio of $n$-heptane to Exxsol D40. Also the measurements are done at different positions due to the limitations of the measurement techniques. The X-Ray evaluation cut is at $400 \mu \mathrm{m}$ distance to the nozzle hole exit. The local spray momentum measurement is done on a sphere with a radius of $10 \mathrm{~mm}$ distance. The calculated force rate out of the local spray momentum measurement and the calculated force rate out of the X-ray measurement are given in Figure 9. Additionally the integrated spray momentum curves are displayed.

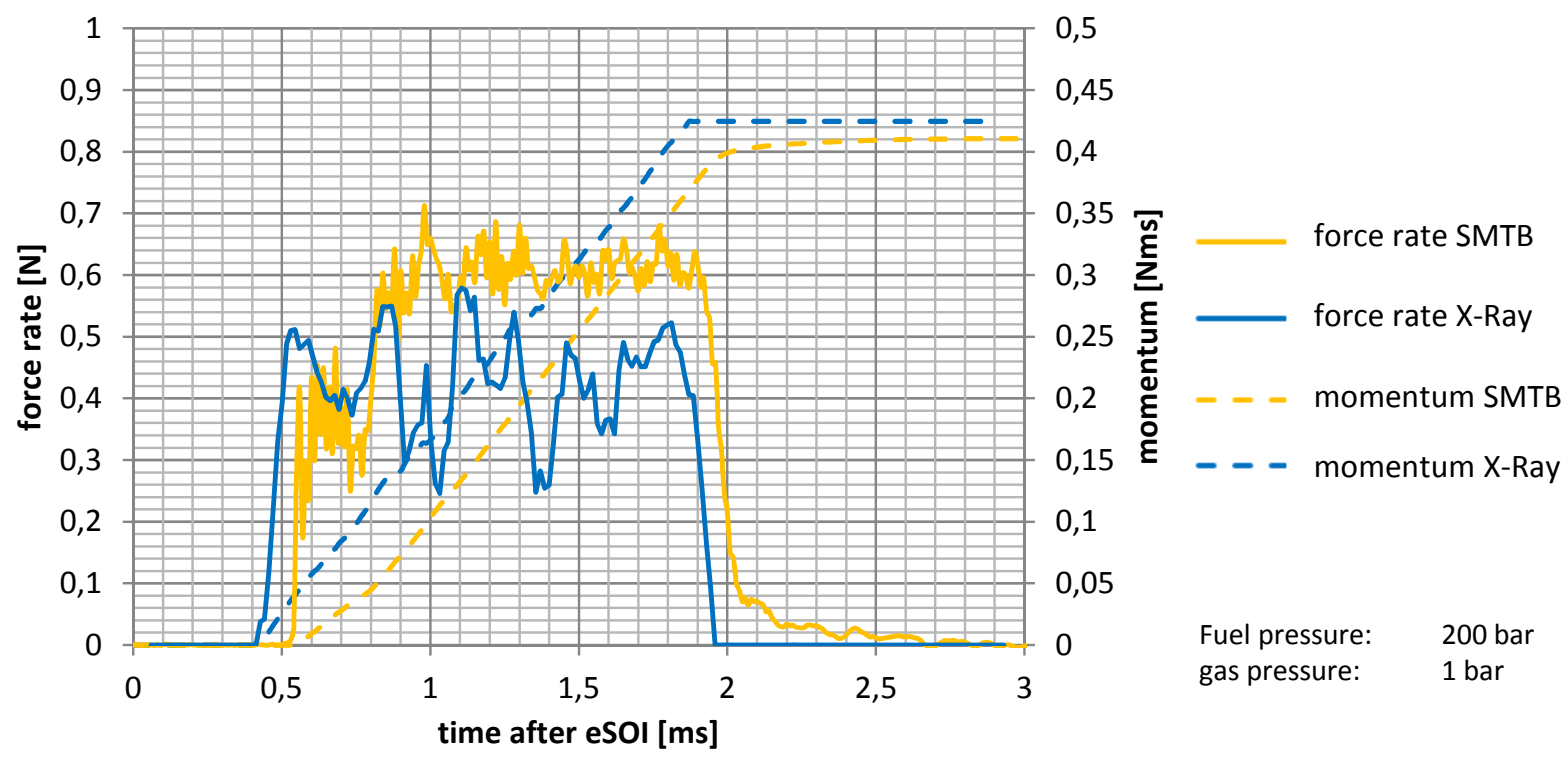

Figure 9. Comparison of the calculated spray momentum of High Speed X-Ray Imaging and the SMTB

The spray force rate of the SMTB is shifted to later points in time as the spray has to pass the distance of $10 \mathrm{~mm}$ before reaching the measurement pin. Also the filtering of the raw spray force signal will result in a jitter. It can be 
seen that both measurement techniques deliver spray momentum values in the same range. The SMTB delivers a slightly smaller spray momentum due to dissipation while traveling the distance to the measurement pin.

\section{Conclusions}

Within this study methods for evaluating velocity and density measurements captured with High Speed X-Ray Imaging are developed. The algorithms are applied exemplary. The results are compared and validated with measurements of a mechanical SMTB. High Speed X-Ray Imaging turns out to be a powerful tool to measure velocity and density distributions inside a GDI spray in the nearfield. The measurement of the density and velocity distributions inside a gasoline spray allow to split up the mechanically easily measurable spray momentum distribution to find the main drivers for momentum differences in different gasoline jets. It will reach its limits in far away distances as the structural velocities and therefore the traveled distances of the structures between two exposures are too small for the very high pulse frequency of the X-ray source.

\section{Acknowledgements}

This work is supported by the US Department of Energy, Basic Energy Sciences, Office of Science, under Contract No. 852 W5.

\section{References}

[1] M. Krämer, E. Kull, Markus Heldmann, M. Wensing. Investigations on gasoline spray porpagation behaviour characteristics for multihole injectors: Sae technical paper 2014-01-2732. 2014.

[2] R. Distler, M. Krämer, E. Kull, M. Wensing. Spray characterization of multihole gdi injectors with spray momentum measurements. Motorische Verbrennung Aktuelle Probleme und moderne Lösungsansätze (XIII. Tagung), 2017.

[3] M. Krämer. Einfluss motorischer Randbedingungen auf die Spraycharakteristik bei direkteinspritzenden Ottoverfahren, volume 2016,2 of Berichte zur Thermodynamik und Verfahrenstechnik. 1. auflage edition, 2016.

[4] M. Krämer, E. Kull, M. Wensing. Flashboiling - induced targeting changes in gdi sprays, 2015.

[5] J. Ernst. Experimentelle und numerische Analyse des Impulses von aufbereitetem Kraftstoffspray im düsennahen Bereich der Dieseleinspritzung. Wissenschftliche Reihe Fahrzeugtechnik Universität Stuttgart. Wiesbaden, 2014.

[6] C. Gerthsen. Gerthsen Physik: Mit 89 Tabellen und 105 durchgerechneten Beispielen und 1065 Aufgaben mit vollständigen Lösungswegen. Springer-Lehrbuch. Springer, Berlin and Heidelberg and New York and Barcelona and Hongkong and London and Mailand and Paris and Singapur and Tokio, 20., aktualisierte aufl. edition, 1999.

[7] Yujie Wang, Xin Liu, Kyoung-Su Im, Wah-Keat Lee, Jin Wang, Kamel Fezzaa, David L.S. Hung, James R. Winkelman. Ultrafast $\mathrm{x}$-ray study of dense-liquid-jet flow dynamics using structure-tracking velocimetry. nature physics, 2008.

[8] A. Kastengren, C. F. Powell. Synchrotron x-ray techniques for fluid dynamics. Exp Fluids, (55:1686), 2014.

[9] P. Cloetens. Contribution to Physe Contrast Imaging, Reconstruction and Tomography with Hard Synchrotron Radiation. 1999.

[10] D. Mukherjee, Sanjoy Das, B.K. Shah. Evaluation of coating layer thickness for simulated triso coated fuel particle by micro focus radiography. Proceedings of the National Seminar \& Exhibition of Non-Destructive Evaluation, 2011. 